\title{
Rubidium Chloride Targets Jnk/p38-Mediated NF-кB Activation to Attenuate Osteoclastogenesis and Facilitate Osteoblastogenesis
}

\author{
Zhengxiao Ouyang ${ }^{1,2}$, Qianli Huang ${ }^{1}$, Bin Liu ${ }^{1}$, Hong Wu ${ }^{1}$, Tang Liu ${ }^{1,2 *}$ and Yong Liu ${ }^{1 *}$ \\ 'State Key Laboratory of Powder Metallurgy, Central South University, Changsha, China, ${ }^{2}$ Department of Orthopedics, \\ The Second Xiangya Hospital, Central South University, Changsha, China
}

OPEN ACCESS

Edited by:

Peter Vee Sin Lee,

The University of Melbourne,

Australia

Reviewed by:

Michaël R. Laurent,

University Hospitals Leuven,

Belgium

Lakshman Singh,

The University of Melbourne,

Australia

*Correspondence:

Tang Liu

liutang1204@csu.edu.cn

Yong Liu

yonliu@csu.edu.cn

Specialty section:

This article was submitted to Translational Pharmacology,

a section of the journal

Frontiers in Pharmacology

Received: 03 February 2019 Accepted: 06 May 2019

Published: 22 May 2019

Citation:

Ouyang Z, Huang Q, Liu B, Wu H, Liu T and Liu Y (2019) Rubidium Chloride Targets Jnk/p38-Mediated $N F-\kappa B$ Activation to Attenuate Osteoclastogenesis and Facilitate Osteoblastogenesis.

Front. Pharmacol. 10:584 doi: 10.3389/fphar.2019.00584
The unbalanced crosstalk between osteoclasts and osteoblasts could lead to disruptive bone homeostasis. Herein, we investigated the therapeutic effects of rubidium chloride $(\mathrm{RbCl})$ on ovariectomized (OVX) and titanium (Ti) particle-induced calvaria osteolysis mouse models, showing that non-toxic $\mathrm{RbCl}$ attenuated RANKL-stimulated osteoclast formation and functionality while significantly enhancing osteogenesis in vitro. The expressions of osteoclast-specific genes were downregulated considerably by $\mathrm{RbCl}$. Despite the direct inhibition of RANKL-induced activation of MAPK signaling, $\mathrm{RbCl}$ was able to target NF- $\mathrm{KB}$ directly and indirectly. We found that after the co-stimulation of the c-Jun $\mathrm{N}$-terminal kinase (Jnk)/p38 activator and RANKL, RbCl inhibited the elevated expression of p-IKK $\alpha$ and the degradation of $\mathrm{I}_{\kappa} \mathrm{B} \alpha$ in osteoclast precursors, indicating indirect $\mathrm{NF}-\kappa \mathrm{B}$ inhibition via MAPK suppression. Furthermore, the two animal models demonstrated that $\mathrm{RbCl}$ attenuated tartrate-resistant acid phosphate (TRAP)-positive osteoclastogenesis and rescued bone loss caused by the hormonal dysfunction and wear particle in vivo. Altogether, these findings suggest that $\mathrm{RbCl}$ can target $\mathrm{Jnk} / \mathrm{p} 38$-mediated NF-kB activation to attenuate osteoclastogenesis, while facilitating osteoblastogenesis both in vivo and in vitro, suggesting the possible future use of $\mathrm{RbCl}$ for surface coating of orthopedic implant biomaterials to protect against osteoporosis.

Keywords: rubidium chloride, osteoclast, osteoblast, MAPK, NF-кB, osteoporosis

\section{INTRODUCTION}

Bone is a dynamically balanced tissue that is consistently regulated by the crosstalk between bone resorption by osteoclasts and bone formation by osteoblasts (Qiao and Tang, 2018; Rao et al., 2018). The concord of osteoclast and osteoblast maintains bone homeostasis via vivid bone remodeling, contributing to the normal physiology of the skeletal system (Seeman and Delmas, 2006). By contrast, the disruption of this equilibrium inevitably results in a series of bone disorders, leading to afflicted skeletal physiology and functionality (Qu et al., 2018). The dysregulated maturation of osteoclasts and inhibited activation of osteoblasts show a considerable morbidity with compromised bone strength and increased bone fragility (Chen et al., 2017; Jing et al., 2018); the condition requires an increasing number of therapeutic remedies. Additionally, it is of interest to uncover novel strategies that combine effects of anti-osteoclastogenesis and pro-osteoblastogenesis simultaneously, which would definitely contribute to the development of an effective treatment against osteoporosis. 
Importantly, the mitogen-activated protein kinase (MAPK) pathway that comprises the c-Jun N-terminal kinase (Jnk), extracellular-signal-regulated kinase (Erk), and p38 has been considered to promote osteoclastogenesis (Liu et al., 2017; Qiao et al., 2017). Likewise, nuclear factor $\kappa B(N F-\kappa B)$ signaling has also been proven to play a crucial role during osteoclast formation (Ouyang et al., 2014; Qiao et al., 2016). Various reports showed the underlying interaction between MAPK and NF- $\kappa B$ (Dhawan and Richmond, 2002; Papa et al., 2004). By activating the $\mathrm{p} 38 / \mathrm{MAPK}$ molecule, NF- $\mathrm{BB}$ appears to emerge via a Cdc42-independent mechanism (Korus et al., 2002). The accumulation of Jnk expression leads to the activation of NF- $\kappa B$ during macroautophagy, resulting in a rescued cancer cell survival (Yang et al., 2017). However, despite numerous studies on osteoporosis that highlight the roles of MAPK and NF- $\kappa B$ during osteoclastogenesis, few of them focus on the connection between MAPK and NF- $\mathrm{BB}$. Therefore, targeting MAPK-mediated NF- $\kappa \mathrm{B}$ activation may serve as a novel strategy in treating osteoporosis with an effective cascade-controlling response.

Considering the crucial roles of MAPK-mediated NF- $\kappa \mathrm{B}$ activation during osteoclastogenesis, we seek to find novel compounds that could inhibit both MAPK and NF- $\kappa B$ activation, thereby effectively attenuating osteoclast formation. Herein, rubidium chloride $(\mathrm{RbCl})$ that normally acts as a perfusion biomarker (Hougardy et al., 2003) and an anti-depressant (Kordjazy et al., 2015) was found to target Jnk/p38 in osteoclast precursors to inhibit osteoclast formation. More importantly, despite significant anti-osteoclast effects, $\mathrm{RbCl}$ could simultaneously promote osteoblastogenesis, thereby promising to satisfactorily treat osteoporosis. Also, few reports have covered the regulatory effects of $\mathrm{RbCl}$ in bone homeostasis. Therefore, we employed $\mathrm{RbCl}$ to provide therapeutic benefits for osteoclast and osteoblast, aiming at re-establishing the homeostasis of the bone microenvironment and elucidating the underlying molecular mechanisms, thus suggesting potential clinical uses of $\mathrm{RbCl}$, such as in surface coating of orthopedic implants.

\section{MATERIALS AND METHODS}

\section{Main Reagents and Cell Culture}

Recombinant macrophage colony stimulating factor (MCSF) and receptor activator for the nuclear factor- $\kappa B$ ligand (RANKL) were purchased from R\&D Systems (Minneapolis, MN, USA). RbCl and fetal bovine serum (FBS) were purchased from Sigma-Aldrich (St. Louis, MO, USA). MAPK activator anisomycin and Asiatic acid were purchased from Sigma-Aldrich. Antibodies for western blotting and immunohistochemical analyses were purchased from Cell Signaling Technology (Cambridge, MA, USA).

Osteoclast precursor RAW 264.7 cells and osteoblast MC3T3-E1 cells were cultured in standard a-MEM medium supplemented with $10 \%$ FBS and $1 \%$ penicillin/streptomycin. Primary murine bone marrow monocytes (BMMs) were harvested from the femurs and tibiae of four- to six-week-old C57BL/6 mice and incubated in a-MEM medium with $30 \mathrm{ng} / \mathrm{ml} \mathrm{MCSF}$ and $10 \%$ FBS. All cells were kept in a sterile condition at $5 \%$ $\mathrm{CO}_{2}$ of $37^{\circ} \mathrm{C}$.

\section{Cell Viability}

The viability of BMMs was assessed with the Cell Counting Kit-8 (CCK-8) method (Luo et al., 2018; Ouyang et al., 2018, 2019a,b; Xiao et al., 2018; Zhang et al., 2018). Briefly, BMMs were first seeded into 96 -well plates at a density of $1.0 \times 10^{5}$ cells/well for $24 \mathrm{~h}$. Next, BMMs were treated with corresponding concentrations of $\mathrm{RbCl}(0,0.1,0.2,0.4,0.8,1.6,3.2,6.4,12.8$, and $25.6 \mathrm{mM}$ ) for up to 4 days. A $10 \mu \mathrm{l}$ CCK-8 solution mixed in $100 \mu \mathrm{l}$ non-serum a-MEM medium was added into plates in $5 \% \mathrm{CO}_{2} 37^{\circ} \mathrm{C}$ for $2 \mathrm{~h}$. Optical density at $450 \mathrm{~nm}$ $\left(\mathrm{OD}_{450}\right)$ was measured.

\section{Osteoclastogenesis in vitro}

BMMs and RAW 264.7 cells seeded in 96-well plates were treated with $\mathrm{RbCl}$ at different concentrations $(0.8,1.6$, and $3.2 \mathrm{mM}$ ). Cell medium with $50 \mathrm{ng} / \mathrm{ml}$ RANKL was changed every other day continuously until the 7 th day. After osteoclast maturation, cells were fixed in $4 \%$ paraformaldehyde (PFA) and then rinsed with PBS. Tartrate-resistant acid phosphatase (TRAP) staining was then deployed to indicate the differentiated multi-nucleated osteoclasts. Stained osteoclasts with at least three nuclei were classified as TRAP-positive osteoclasts (Zhai et al., 2014).

\section{F-Actin Ring Immunofluorescence}

F-actin ring was observed to indicate the ruffled membrane of osteoclasts (Xiao et al., 2015). Differentiated BMMs treated with RANKL $(50 \mathrm{ng} / \mathrm{ml})$ and $\operatorname{RbCl}(0.8,1.6$, and $3.2 \mathrm{mM}$ ) were fixed in $4 \%$ PFA and permeabilized in $0.1 \%$ Triton X-100. Alexa Fluor 647 phalloidin was then used to stain the osteoclast cytoskeleton. DAPI indicated cell nuclei. After several rinses with PBS, the fluorescent F-actin ring was observed under confocal microscopy (Leica TCS SP8, Germany).

\section{Bone Resorption Pit Evaluation}

BMMs were seeded onto bovine bone slices at a density of 7,000 cells/slice in complete $\alpha$-MEM medium with $\mathrm{RbCl}(0.8,1.6$, and $3.2 \mathrm{mM}$ ), RANKL (50 $\mathrm{ng} / \mathrm{ml})$, and MCSF (50 $\mathrm{ng} / \mathrm{ml})$. After the formation of matured osteoclasts, the adherent cells were removed by mechanical agitation and sonication. Scanning electron microscopy (SEM) was then used to visualize the osteolytic resorption pit.

\section{Quantitative Real-Time PCR}

After treatment with $\mathrm{RbCl}(3.2 \mathrm{mM})$ and RANKL for 7 days, the BMMs seeded in 6-well plates were harvested to extract total RNA using RNeasy Mini Kit (Qiagen, CA, USA) following the manufacturer's instructions. cDNA was synthesized from extracted RNA with reverse transcriptase kit (Takara Biotechnology, Japan). Real-time PCR was performed with SYBR Premix Ex Taq Kit (Takara Biotechnology, Japan) using ABI 7500 Sequencing Detection System (Applied Biosystems, Foster City, CA, USA), according to protocol under deltadelta-CT method. Mouse primers for CTR, CtsK, NFATc-1, TRAP, and GAPDH were listed as follows: CTR forward 5'-TGCAGACAACTCTTGGTTGG-3' and reverse 
5' -TCGGTTTCTTCTCCTCTGGA-3'; CtsK forward 5' -CTTCCA ATACGTGCAGCAGA- $3^{\prime}$ and reverse $5^{\prime}$-TCTTCAGGGC TTTCTCGTTC-3'; NFATc-1 forward 5'-CCGTTGCTTCC AGAAAATAACA- $3^{\prime}$ and reverse $5^{\prime}$-TGTGGGATGTGAACT CGGAA-3'; TRAP forward 5'-CTGGAGTGCACGATGCCAGCGACA-3' and reverse $5^{\prime}$-TCCGTGCTCGGCGATGG ACCAGA-3'; GAPDH forward 5'-ACCCAGAAGACTGTGGA TGG-3' and reverse $5^{\prime}$-CACATTGGGGGTAGGAACAC-3'.

\section{Western Blotting}

RAW 264.7 cells seeded in 6-well plates were pre-treated with or without $12.8 \mathrm{mM} \mathrm{RbCl}$ for $4 \mathrm{~h}$. Afterward, $50 \mathrm{ng} / \mathrm{ml}$ RANKL was administered at specific time points $(0,5,10$, 20, 30, and $60 \mathrm{~min})$ to determine the RANKL-activated expression of MAPK and NF- $\kappa B$. Total protein was harvested with radioimmunoprecipitation assay (RIPA) lysis buffer (Beyotime Bioscience, Shanghai, China) supplemented with $1 \mathrm{mM}$ phenylmethylsulfonyl fluoride (PMSF) and quantified with a BCA Protein Assay Kit (Thermo Scientific, IL, USA). Lysates were diluted in a loading buffer and denatured at $99^{\circ} \mathrm{C}$ for $10 \mathrm{~min}$. Thirty micrograms of lysate were first resolved in sodium dodecyl sulfate-polyacrylamide gel electrophoresis (SDS-PAGE) on $10 \%$ precast gel at $80 \mathrm{~V}$ for $30 \mathrm{~min}$ and $120 \mathrm{~V}$ for $1 \mathrm{~h}$, and then transferred to activated polyvinylidene fluoride (PVDF) membrane for $2.5 \mathrm{~h}$ at $250 \mathrm{~mA}$. Five percent non-fat milk powder dissolved in Tris-buffer saline containing $0.05 \%$ Tween (TBST) was used to block membrane, and the first antibody was administered overnight at $4^{\circ} \mathrm{C}$. After several rinses with TBST, the membrane was probed with corresponding secondary antibody for $1 \mathrm{~h}$, followed by the visualization of protein bands using the Odyssey Infrared Imaging System (LI-COR Bioscience, NE, USA).

\section{Luciferase Reporter Gene Activity Assay}

Since NFATc-1 served as downstream molecules of NF- $\kappa B$ and MAPK (Jiang et al., 2015), we employed NFATc-1 luciferase reporter gene assay on the basis of RAW264.7 cells that stably transfected with NFATc-1 luciferase reporter constructs, to determine the inhibitory effects of RbCl. Transfected RAW264.7 cells were treated with $\mathrm{RbCl}(1.6,3.2 \mathrm{mM})$ and RANKL for $7 \mathrm{~h}$, and luciferase activities were investigated using a Promega Luciferase Assay System (Madison, WI, USA) following the manufacturer's instructions.

\section{Molecular Docking Assay}

Using the architectures of human Jnk/p38 as templates, we constructed three-dimensional homology models of mouse Jnk/p38 kinase domains with Modeler 9.12. On the basis of AutoDock and AutoDock Vina (Trott and Olson, 2010), PROCHECK was used to verify the stereochemical structures of Jnk/p38, while Lamarckian genetic algorithm was utilized to link $\mathrm{RbCl}$ with kinases. The final figures of molecular docking indicating binding activity were presented with the PyMOL Visualization Software (Schrödinger LLC, New York, NY, USA).

\section{Alkaline Phosphatase (ALP) Staining and Quantification}

Osteoblast MC3T3-E1 cells were seeded in 24-well plates at a density of $5.0 \times 10^{4} \mathrm{~cm}^{-2}$ and treated with an osteogenic inductive medium including $100 \mathrm{nM}$ dexamethasone, $50 \mu \mathrm{g} / \mathrm{ml}$ ascorbic acid, $10 \mathrm{mM} \beta$-glycerophosphate sodium, and different concentrations of $\mathrm{RbCl}$ (low: $1.6 \mathrm{mM}$; high: $3.2 \mathrm{mM}$ ). The medium was refreshed every 2 days until the 14th day. ALP staining was performed according to previously reported procedures (Tan et al., 2012), and ALP activity was determined using an ALP microplate test kit (Beyotime Bioscience, Shanghai, China), with the absorbance recorded at an optical density of $520 \mathrm{~nm}\left(\mathrm{OD}_{520}\right)$. The results were normalized to the corresponding total protein content using a BCA Protein Assay Kit (Thermo Scientific, IL, USA).

\section{Alizarin Red S (ARS) Staining and Quantification}

Osteoblast MC3T3-E1 cells were seeded in 24-well plates at a density of $5.0 \times 10^{4} \mathrm{~cm}^{-2}$ and treated with an osteogenic inductive medium including $100 \mathrm{nM}$ dexamethasone, $50 \mu \mathrm{g} / \mathrm{ml}$ ascorbic acid, $10 \mathrm{mM} \beta$-glycerophosphate sodium, and different concentrations of $\mathrm{RbCl}$ (low: $1.6 \mathrm{mM}$; high: $3.2 \mathrm{mM}$ ). The medium was refreshed every 2 days until the 21 st day. Cells were then fixed in $4 \% \mathrm{PFA}$ for $15 \mathrm{~min}$ at $37^{\circ} \mathrm{C}$ before being stained with $1 \%$ ARS solution (Sigma-Aldrich) for $50 \mathrm{~min}$. PBS was used to wash the non-specific staining, and representative images were taken by optical microscopy. For the quantitative assay, $10 \%$ cetylpyridinium chloride in $10 \mathrm{mM}$ sodium phosphate was administered to stained cells, followed by the determination of optical density at $620 \mathrm{~nm}\left(\mathrm{OD}_{620}\right)$.

\section{Ovariectomized (OVX) Mouse Model}

This study was carried out in accordance with the recommendations of guiding principles of Animal Care Committee of Central South University, and the protocol was approved by the Animal Care Committee of Central South University. Eight-week-old C57BL/6 mice $(n=24)$ were obtained from Shanghai Slac Laboratory Animal Company (Slac, Shanghai, China). Mice were kept in plastic-isolator cages under specific pathogen-free (SPF) conditions for 7 days to acclimatize, before OVX surgery was performed on them. Based on previously described procedures (Liu et al., 2017), mice were administered with intraperitoneal ketamine and xylazine mixture for anesthetization and analgesia. Next, a retroperitoneal incision was made ventral to the erector spinae muscles just caudal to the last rib to provide access to the ovary. The ovary was removed with catgut ligature located around the cranial portion of the uterus and uterine vessels, after which the incision was closed and mice were transported to warm conditions for recovery. After ovary resection, mice were randomly assigned into four groups: Sham (non-OVX mice), Vehicle (OVX mice), low (OVX mice injected with $4.5 \mathrm{mg} / \mathrm{kg}$.bw $\mathrm{RbCl}$ intraperitoneally, three times/week), and high (OVX mice injected with $9 \mathrm{mg} / \mathrm{kg}$.bw $\mathrm{RbCl}$ intraperitoneally, three times/week; $n=6$ for each group; the doses of $\mathrm{RbCl}$ used were screened after 
preliminary selections in vivo). Sham and Vehicle mice received an injection of sterile PBS three times a week. Mice were injected and observed for 4 weeks.

\section{Titanium (Ti) Particle-Induced Calvarial Osteolysis Model}

We also used a mouse calvarial osteolysis model to assess the effects of $\mathrm{RbCl}$ as described previously (Ouyang et al., 2014). Eight-week-old C57BL/6 mice $(n=24)$ were obtained from Shanghai Slac Laboratory Animal Company and kept in plasticisolator cages under SPF condition for 7 days to acclimatize, before model establishment. Mice were administered with intraperitoneal ketamine for anesthetization, and the cranial periosteum was separated from calvarium by sharp dissection. Afterward, titanium particles $(30 \mathrm{mg}$ ) were imbedded under the periosteum at the central suture of the calvaria. After $\mathrm{Ti}$ particle embedment, mice were randomly assigned into four groups and received drug administrations as mentioned above for the OVX model. Mice were injected and observed for 10 days.

\section{$\mu$ CT Scanning}

After treatment with $\mathrm{RbCl}$, mice were euthanized, and the harvested tibiae and calvaria were fixed in 4\% PFA before being transferred into a $\mu \mathrm{CT}$ scanning ( $\mu \mathrm{CT}$ 40, Scanco, Zurich, Switzerland). The parameters used for the $\mu \mathrm{CT}$ tomography at a resolution of $10 \mu \mathrm{m}$ were $80 \mathrm{kV}$ (X-ray voltage) and $80 \mu \mathrm{A}$ (electric current). Relevant bone volume fractions (BV/TV, \%), trabecular number (Tb.N, 1/mm), trabecular separation ( $\mathrm{Tb} . \mathrm{Sp}, \mathrm{mm}$ ), and trabecular thickness (Tb.Th, mm) were obtained to analyze the effects of $\mathrm{RbCl}$ on OVX mice. The region of interest (ROI) in proximal tibia was defined to cover the whole subchondral bone in tibiae plateaus. Furthermore, the square ROI around the midline suture of the calvaria was selected, and relevant bone volume fractions (BV/TV, \%), number of porosities, and the

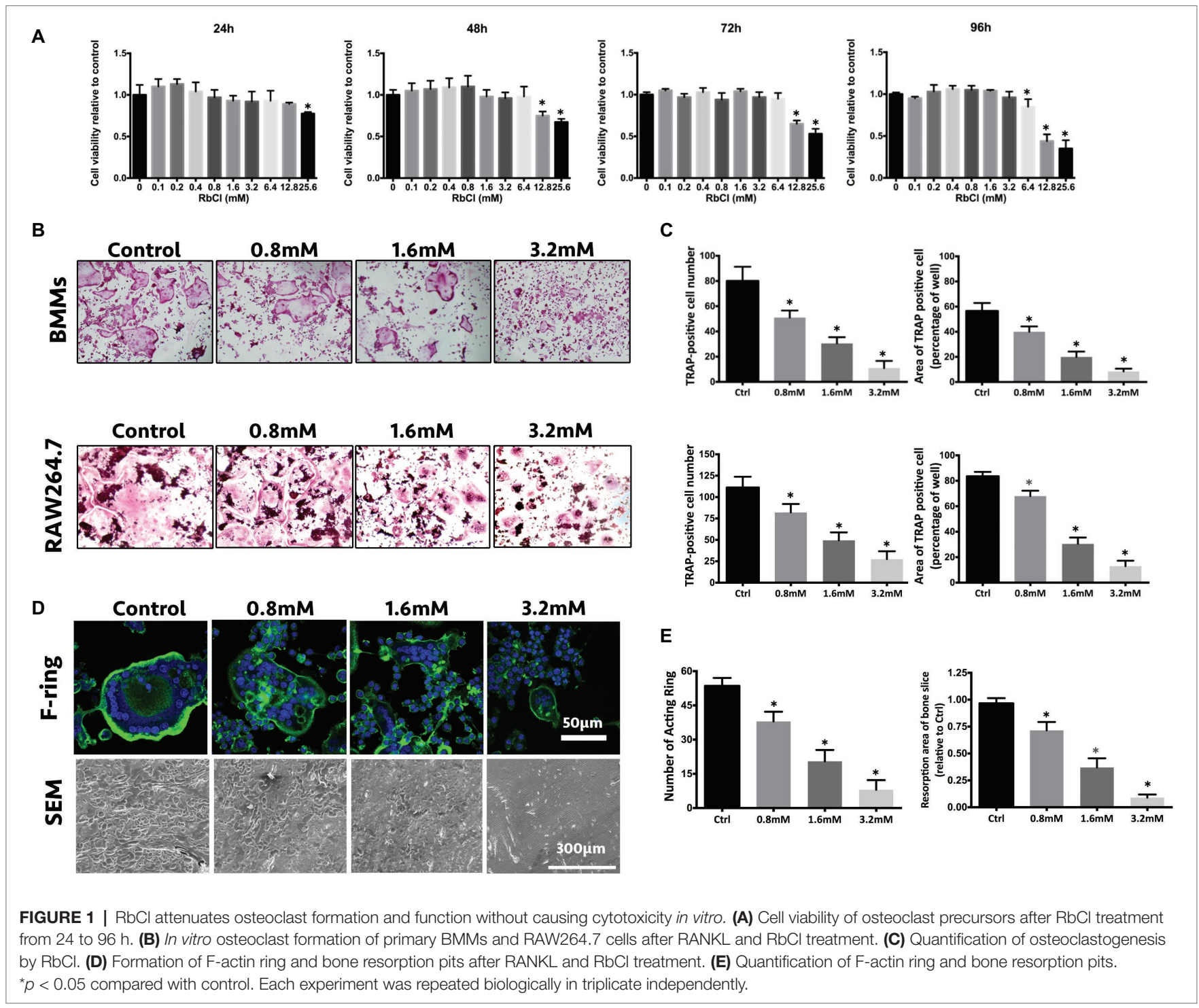


percentage of total porosities (\%) were measured to evaluate the effects of $\mathrm{RbCl}$ on wear-particle induced osteolysis mice.

\section{Immunohistochemical Staining Analyses}

After $\mu \mathrm{CT}$ analysis, harvested tibiae and calvaria were decalcified in $10 \%$ EDTA for 4 weeks, followed by paraffin-embedded procedures. Tissues were sectioned and stained for both hematoxylin-eosin (HE) and TRAP solution following the manufacturer's guidelines.

\section{Statistical Calculations}

All the data acquired from both in vitro and in vivo experiments were presented as means \pm standard deviations (SD) and analyzed with SPSS 13.0 software (SPSS Inc., USA), using one-way analysis of variance. A two-tailed $p<0.05$ was considered as statistically significant. All the experiments were repeated biologically at least three times independently.

\section{RESULTS}

\section{$\mathrm{RbCl}$ Attenuates Osteoclast Formation and Function in vitro Without Causing Cytotoxicity}

To investigate the inhibitory effects of $\mathrm{RbCl}$ on osteoclast formation and function, we first sought to explore the non-toxic concentrations of $\mathrm{RbCl}$ upon osteoclast precursors and to exclude the direct suppressive effects upon osteoclast proliferation of $\mathrm{RbCl}$. As shown in Figure 1A, only the highest concentration of $\mathrm{RbCl}(25.6 \mathrm{mM})$ showed significant osteoclast inhibitory effects after $24 \mathrm{~h}$. With the increase of incubation time, it was evident that $25.6,12.8$, and $6.4 \mathrm{mM} \mathrm{RbCl}$ were able to exert significant osteoclast inhibition after $96 \mathrm{~h}$, suggesting a dose-dependent action of $\mathrm{RbCl}$ on osteoclast proliferation. Therefore, non-toxic $\mathrm{RbCl}$ of $0.8,1.6$, and $3.2 \mathrm{mM}$ were selected for further anti-osteoclastogenesis assessment. We found that for BMMs and RAW 264.7 cells, despite significant osteoclast formation showing large, round, and red-stained multinucleated osteoclasts in Ctrl, $\mathrm{RbCl}$ treatments decreased the number and area of the matured TRAP-positive osteoclasts dose-dependently (Figures 1B,C), signifying that $\mathrm{RbCl}$ could attenuate osteoclast formation significantly, without causing cytotoxicity.

Furthermore, since the formation of a well-shaped F-actin ring is indispensable for osteoclast functionality, the regulatory effects of $\mathrm{RbCl}$ on F-actin formation were also investigated. It was shown that in the control group, RANKL administration stimulated osteoclast to develop characteristically polarized F-actin ring. However, $\mathrm{RbCl}$-mediated intervention led to a dramatic decrease in the F-actin rings, in terms of size and number (Figures 1D,E), implying that osteoclast functionality was damaged by the $\mathrm{RbCl}$ treatments. As expected, large and deep bone resorption pits were formed on bone slices of BMMs upon RANKL stimulation. Conversely, treatment with $\mathrm{RbCl}$ decreased both the number and the

\section{CTR}

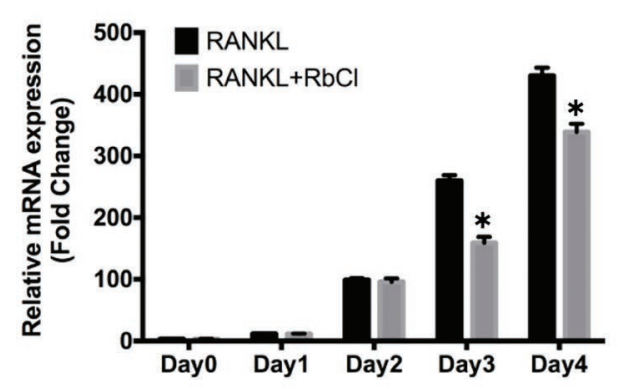

NFATc1

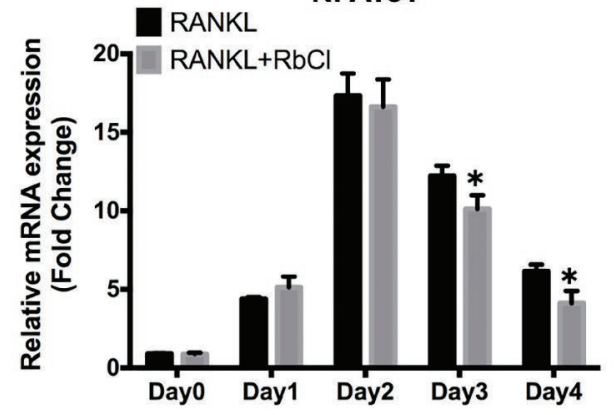

CtsK

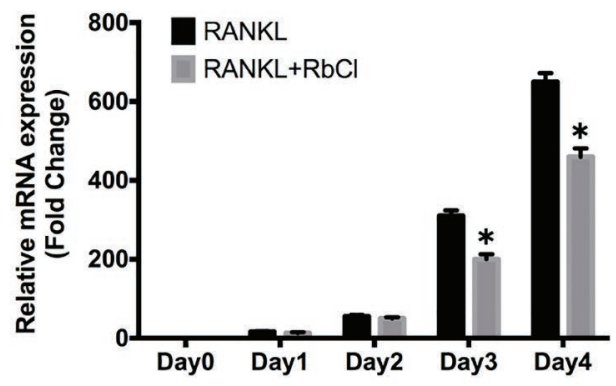

TRAP

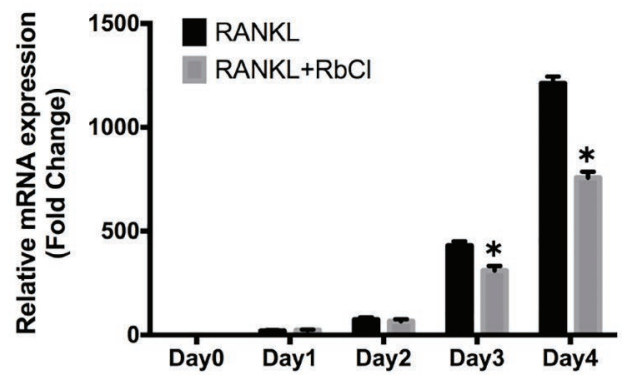

FIGURE 2 | RbCl impairs RANKL-induced osteoclast gene expression. Evaluation of mRNA levels of osteoclast-specific genes in osteoclast precursors. ${ }^{*} p<0.05$ compared with RANKL-treatment only. Each experiment was repeated biologically in triplicate independently. 
area of bone resorption pits on bone slices, showing the potential suppressive effects of $\mathrm{RbCl}$ on osteoclast formation and functionality in vitro.

\section{$\mathrm{RbCl}$ Impairs RANKL-Induced Osteoclast Gene Expression}

During osteoclast formation, RANKL stimulates the expression of particular genes in osteoclasts, which lead to the differentiation from osteoclast precursors to matured osteoclasts (Boyle et al., 2003). As shown in Figure 2, it was evident that along with the progress of osteoclast differentiation, expression of osteoclastic markers CTR, CtsK, and TRAP increased steadily after RANKL stimulation. However, in comparison with RANKL-treatment only, the cells treated with $\mathrm{RbCl}$ and RANKL showed significantly decreased expression of CTR, CtsK, and TRAP. Furthermore, despite upregulation of NFATc-1 level in the first 2 days after RANKL administration, no statistical difference was seen after treatment with $\mathrm{RbCl}$ and RANKL. By contrast, the expression of NFATc-1 decreased during the following 2 days, indicating a significant inhibition of NFATc- 1 by $\mathrm{RbCl}$ compared with the control. These results demonstrated that treatment with
$\mathrm{RbCl}$ attenuated the production of osteoclast-specific genes during osteoclastogenesis in vitro.

\section{RbCl Suppresses RANKL-Induced Jnk/p38-Mediated NF-кB Co-activation}

We aimed to unravel the effects of $\mathrm{RbCl}$ on RANKL-induced MAPK and NF- $\mathrm{BB}$ co-activation during osteoclast formation, thereby inhibiting the expression of osteoclast-specific genes.

As shown in Figure 3A, RANKL induced the increase of p-Erk at $5,10,20$, and $30 \mathrm{~min}$ compared with that at $0 \mathrm{~min}$, while $\mathrm{RbCl}$ delayed this tendency, as evidenced by the expression of p-Erk at 10, 20, and 30 min after $\mathrm{RbCl}$ treatment. Interestingly, we found that Jnk and p38 were steadily phosphorylated after RANKL stimulation, which was significantly attenuated after $\mathrm{RbCl}$ administration, indicating that $\mathrm{RbCl}$ could target phosphorylation of Jnk and p38, thereby inhibiting RANKLstimulated MAPK activation.

Based on the above results, we attempted to determine the potential binding sites between $\mathrm{RbCl}$ with Jnk/p38 kinases by molecular docking analysis. As expected, results showed that $\mathrm{RbCl}$ could be embedded into the binding pockets of both

\section{A}

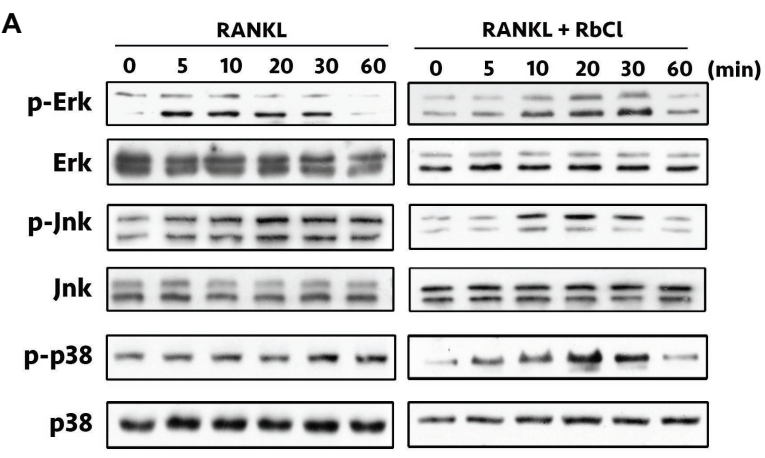

c
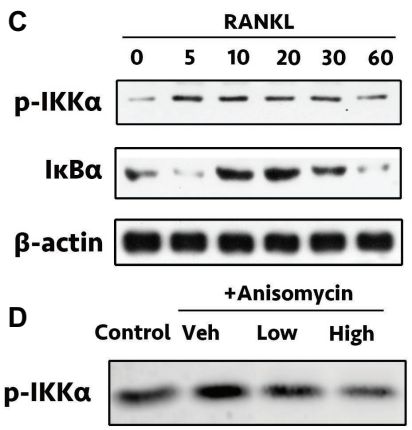

ІкBa

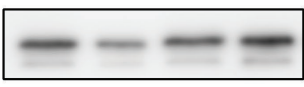

$\beta$-actin
RANKL + RbCl
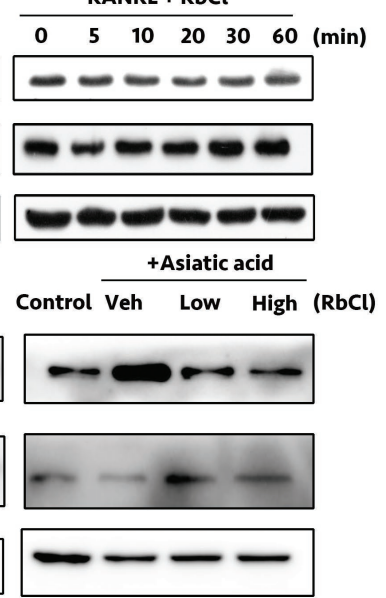

B

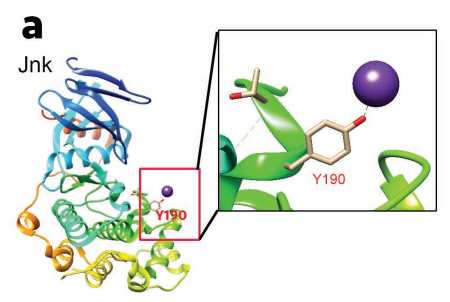

b

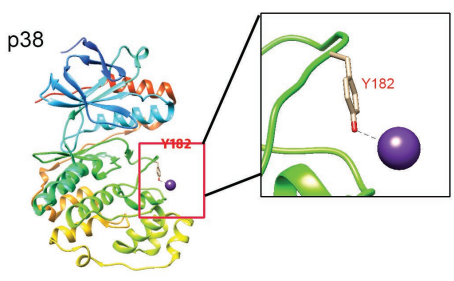

E

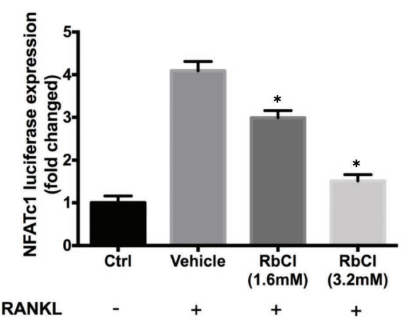

FIGURE 3 | RbCl suppresses RANKL-induced Jnk/p38-mediated NF-kB co-activation. (A) Evaluation of suppressive effects on RANKL-stimulated MAPK signaling by RbCl. (B) Molecular docking of RbCl with Jnk/p38 kinases. (C) Evaluation of inhibitory effects on RANKL-induced NF-kB signaling by RbCl. (D) Transfected RAW264.7 cells were incubated with RbCl and RANKL, and luciferase activity for NFATc-1 was evaluated and normalized to the control. (E) Assessment of NF-kB activation after treatment with anisomycin (Jnk/MAPK activator), Asiatic acid (p38/MAPK activator), RANKL (50 ng/ml), and RbCl (low: 1.6 mM; high: 3.2 mM). ${ }^{*} p<0.05$ compared with Vehicle. Each experiment was repeated biologically in triplicate independently. 
Jnk and p38 kinases by establishing molecular bonds with tyrosine 190 and 182, respectively (Figure 3B). These results indicated that by targeting Jnk and p38 kinases, $\mathrm{RbCl}$ could possibly inhibit RANKL-induced MAPK activation, thereby attenuating osteoclast formation.

It was shown that constitutive activation of MAPK was associated with the increased phosphorylation of NF- $\kappa B$ signaling (Dhawan and Richmond, 2002; Korus et al., 2002; Papa et al., 2004), demonstrating the latent crosstalk between NF- $\kappa B$ and MAPK. Herein, we found that compared with 0 min of RANKL stimulation, the expression of $\mathrm{p}$-IKKa increased within $30 \mathrm{~min}$ and decreased at $60 \mathrm{~min}$. However, this tendency was invalidated by $\mathrm{RbCl}$, which showed no significant differences $60 \mathrm{~min}$ after RANKL administration. Furthermore, I $\mathrm{B}$ a $\alpha$ was significantly degraded at 5 and $60 \mathrm{~min}$, signifying the potent activity of I $\mathrm{B} \alpha$ after RANKL stimulation. Conversely, $\mathrm{RbCl}$ treatment inhibited the degradation of $\mathrm{I} \kappa \mathrm{B} \alpha$, resulting in the significant inactivation of the NF- $\kappa \mathrm{B}$ pathway (Figure $3 \mathrm{C}$ ).

Further, anisomycin (Jnk/MAPK activator) and Asiatic acid (p38/MAPK activator) were used to administer RANKLstimulated osteoclasts to uncover the underlying connection between NF- $\kappa \mathrm{B}$ and MAPK during osteoclastogenesis after $\mathrm{RbCl}$ treatments. We found that despite the activation of $\mathrm{p}-\mathrm{IKKa}$ and I $\kappa \mathrm{B} \alpha$ after activator/RANKL administration, $\mathrm{RbCl}$ inhibited $\mathrm{p}-\mathrm{IKK} \alpha$ and increased I $\mathrm{BB} \alpha$ expression significantly in osteoclasts (Figure 3D). Such results confirmed our hypothesis, that by targeting Jnk/p38-mediated $\mathrm{NF}-\mathrm{\kappa B}$ activation, $\mathrm{RbCl}$ tends to attenuate RANKL-induced osteoclastogenesis significantly, in vitro.

Next, since $\mathrm{RbCl}$ inactivated RANKL-induced MAPK and $N F-\kappa B$, we aimed to unravel the alteration of downstream molecules (NFATc-1) of MAPK and NF- $\kappa B$ (Jiang et al., 2015). Herein, luciferase assay was deployed, showing that NFATc-1 was increased after RANKL administration. However, both low and high concentrations of $\mathrm{RbCl}$ tended to attenuate the activity of NFATc-1 dose-dependently (Figure 3E), signifying that $\mathrm{RbCl}$ inhibited the levels of NFATc-1 after targeting upstream NF- $\kappa B$ and MAPK during osteoclast formation.

\section{$\mathrm{RbCl}$ Promotes Osteoblastogenesis in vitro}

ALP, ARS, and relative quantitative analysis were performed to comprehensively understand the effects of $\mathrm{RbCl}$ on

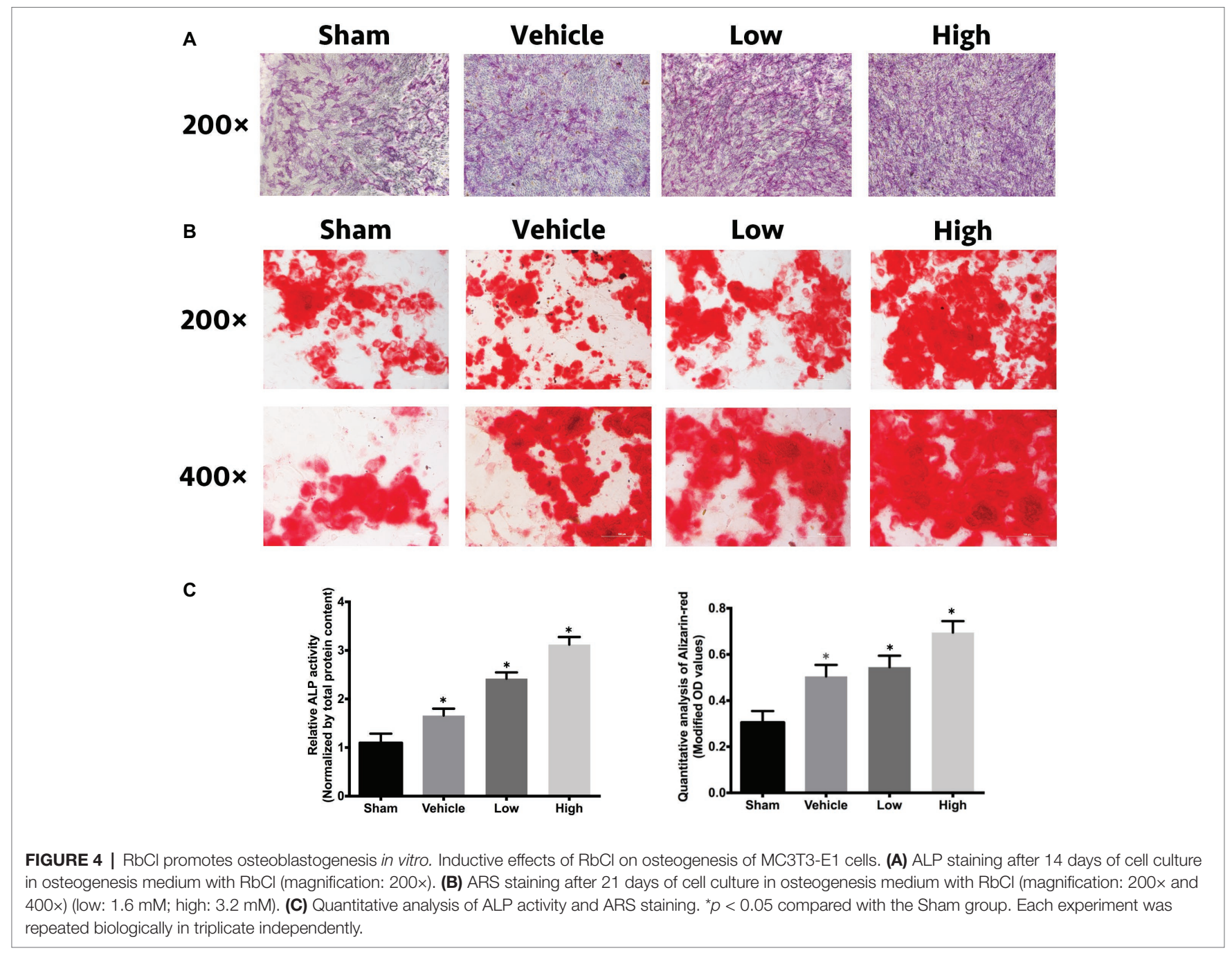


osteoblastogenesis. Figures $\mathbf{4 A - C}$ showed that in comparison with Sham, the intensity of ALP and ARS in Vehicle was considerably stronger. Also, the intensities of osteoblastogenesis after $\mathrm{RbCl}$ treatment were even higher than those of Vehicle, signifying that $\mathrm{RbCl}$ could exhibit significant osteogenesis inductive effects despite its anti-osteoclastogenesis ability, which may provide a promising toolkit for the treatment of osteoporosis.

\section{RbCl Inhibits Bone Loss in OVX Mice}

After the evaluation of the effects of $\mathrm{RbCl}$ on osteoclasts and osteoblasts in vitro, the OVX mice model was employed to investigate the treatment effects of $\mathrm{RbCl}$ in vivo. As shown in Figure $\mathbf{5 A}, \mu \mathrm{CT}$ showed an evident bone loss in Vehicle mice (OVX model). However, treatments of $\mathrm{RbCl}$ in vivo tended to attenuate such tendency, as exemplified by the better-preserved trabecular structure and bone density. Figure 5B of bone histomorphometric evaluation further demonstrated that compared with the Sham group, BV/TV, Tb.N, and Tb.Th decreased while Tb.Sp increased in the Vehicle group, showing osteoporosis phenotype in vivo. By contrast, the values of BV/TV, Tb.N, and
Tb.Th increased, whereas $\mathrm{Tb} . \mathrm{Sp}$ decreased after $\mathrm{RbCl}$ administration, implying that $\mathrm{RbCl}$ could inhibit bone loss in OVX mice.

Also, immunohistochemical analyses (Figure 5C) of $\mathrm{HE}$ staining showed the sparse trabecular architecture in tibiae in Vehicle in comparison with Sham, which was rescued by the administration of $\mathrm{RbCl}$ with intact knee joint and dense trabecular microarchitecture. Furthermore, increased intensity of TRAP-positive osteoclasts was observed in the Vehicle group, compared with that in Sham. However, $\mathrm{RbCl}$ downregulated the number of TRAP-positive osteoclasts (Figure 5D), showing the preservation of bone mass that resulted in the potential preservation of $\mathrm{RbCl}$ against bone loss in vivo.

\section{RbCl Suppresses Ti Particle-Induced Osteolysis in vivo}

In addition to OVX mice, we also used another osteolysis model to assess the effects of $\mathrm{RbCl}$ against osteoclastogenesis. As shown in Figure 6A, in comparison with Sham mice, a large area of osteolysis was observed in the Vehicle group. After $\mathrm{RbCl}$ treatment, $\mathrm{Ti}$ particle-induced bone resorption was inhibited considerably in a dose-dependent manner,

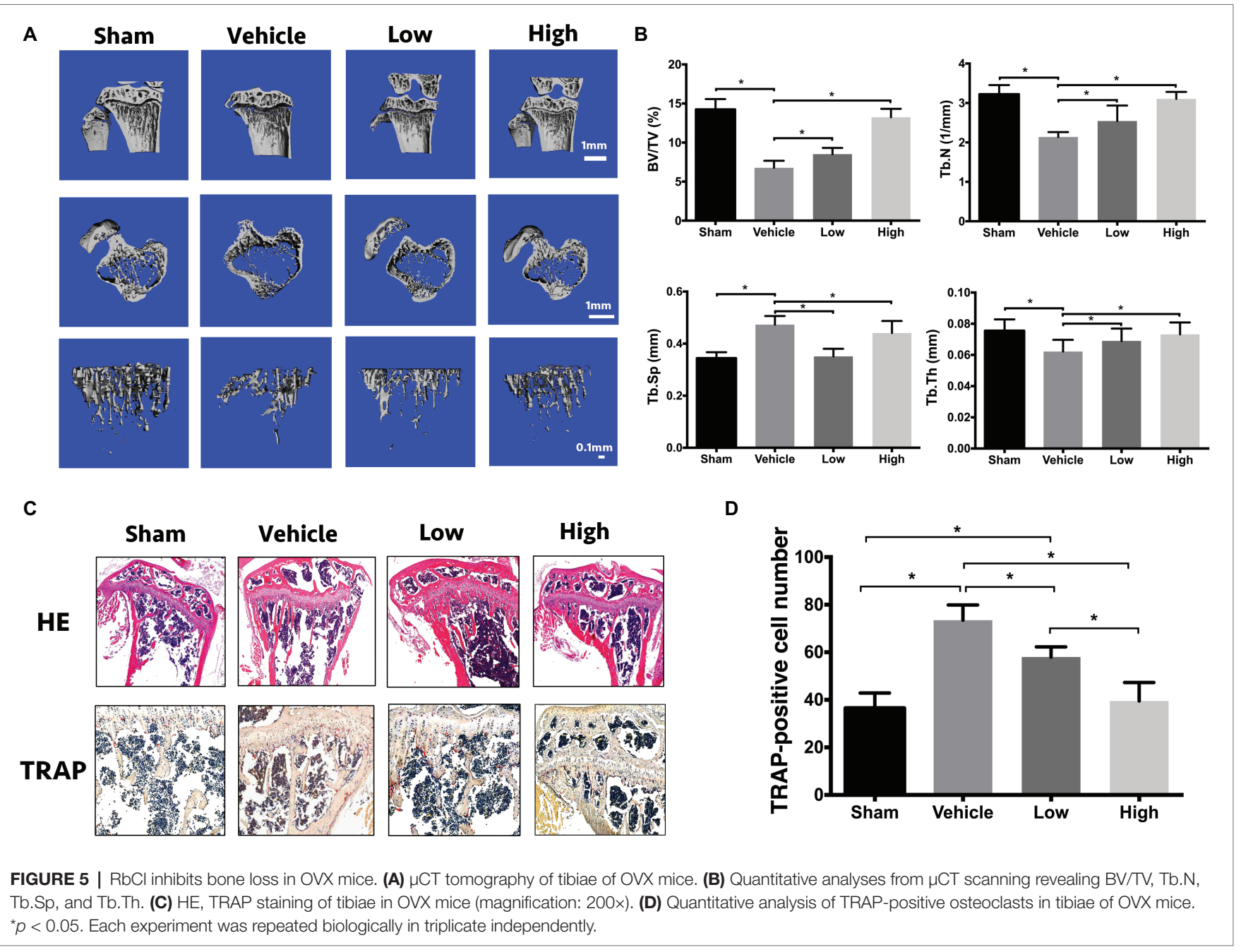


implicating the significant suppressive effects of $\mathrm{RbCl}$ upon the formation of particle-induced osteolytic lesions. Besides, within the ROI area of calvaria, Ti particle induced significant decrease in $\mathrm{BV} / \mathrm{TV}$ and increase in number/percentage of porosity (Figure 6B), which was abrogated by the treatments of $\mathrm{RbCl}$ in varying level compared with that in the Sham group, indicating the potential protective effects of $\mathrm{RbCl}$ against bone loss caused by $\mathrm{Ti}$ particle in vivo.
Furthermore, based on immunohistochemical analyses, we found that the administration of $\mathrm{Ti}$ particles induced the infiltration of lymphocytes and macrophages into calvaria significantly (Figure 6C). However, such inflammation was inhibited by the administration of $\mathrm{RbCl}$, showing the alleviation of inflammatory infiltration and intact trabecular microstructure. Also, TRAP staining illustrated the alignment of mature multinucleated osteoclasts around eroded bone lesions in Vehicle.

A

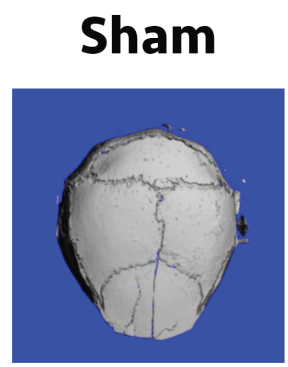

Vehicle

Low

High
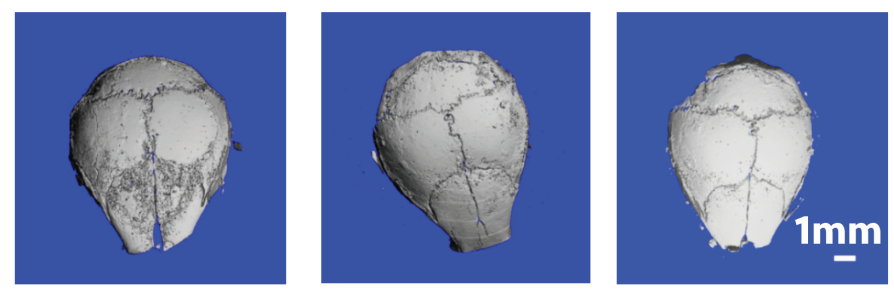

B
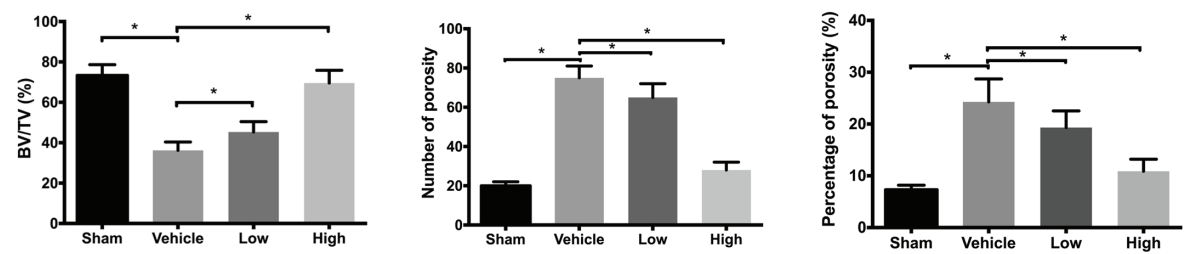

C
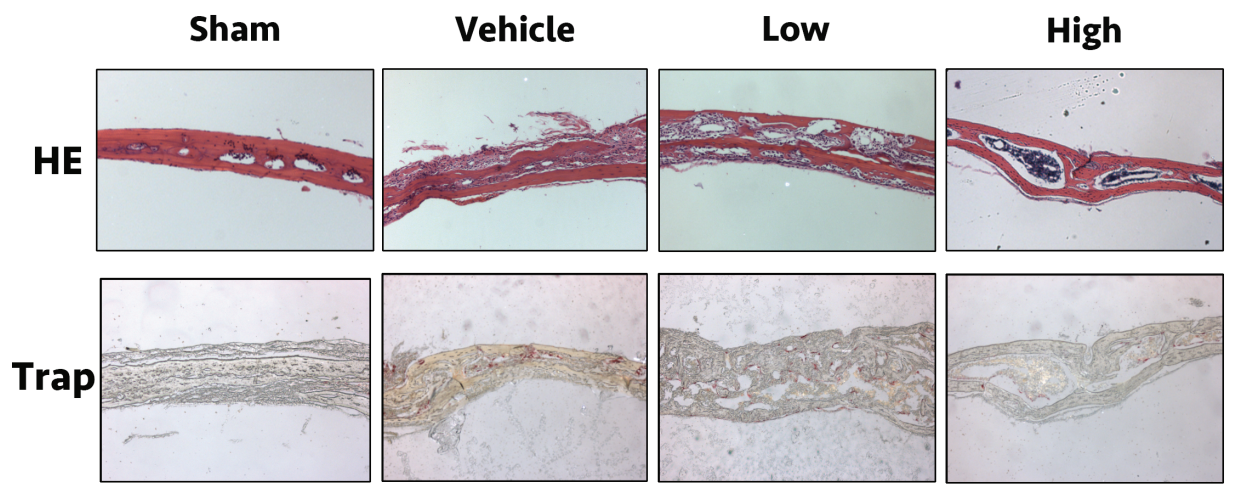

D

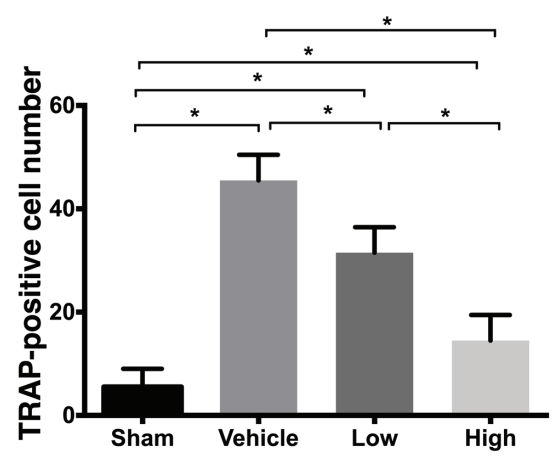

FIGURE 6 | RbCl suppresses Ti particle-induced osteolysis in vivo. (A) $\mu \mathrm{CT}$ tomography of calvaria in Ti particle-induced osteolysis mice model. (B) Quantitative analyses from $\mu$ CT scanning revealing BV/TV, number/percentage of porosity. (C) HE, TRAP of calvaria in Ti particle-induced osteolysis mice (magnification: 200x). (D) Quantitative analysis of TRAP-positive osteoclasts in calvaria in Ti particle-induced osteolysis mice. ${ }^{*} p<0.05$. Each experiment was repeated biologically in triplicate independently. 
$\mathrm{RbCl}$ treatments reduced the area of osteolytic lesions significantly, with a diminished number of TRAP-positive osteoclasts in calvaria osteolysis (Figure 6D). Collectively, in vivo mice model showed that $\mathrm{RbCl}$ inhibited bone loss caused by hormone dysfunction and Ti particles significantly, indicating the strong possibility for future clinical translation of $\mathrm{RbCl}$, such as in the surface coating of orthopedic implants.

\section{DISCUSSION}

The vivid crosstalk between MAPK and NF-kB has long been understood. However, current research seems insufficient to utilize this connection to inhibit osteoclast formation. Besides, in the condition of osteoporosis, it is of significance to attenuate osteoclastogenesis while facilitating osteoblastogenesis simultaneously. Herein, we have demonstrated that $\mathrm{RbCl}$ could inhibit RANKL-induced osteoclast formation and promote MC3T3-E1 cell osteoblastogenesis. Besides, by targeting Jnk/ p38 molecules of MAPK signaling, $\mathrm{RbCl}$ not only inhibited the activation of RANKL-stimulated MAPK pathway but also ameliorated Jnk/p38-mediated NF- $\mathrm{KB}$ activation, thus providing potent inhibitory effects against osteoclastogenesis. (Figure 7) Also, on the basis of the two osteolysis animal models, we have shown the effective inhibition of $\mathrm{RbCl}$ against osteolysis induced by hormonal dysfunction and wear particles, extending the

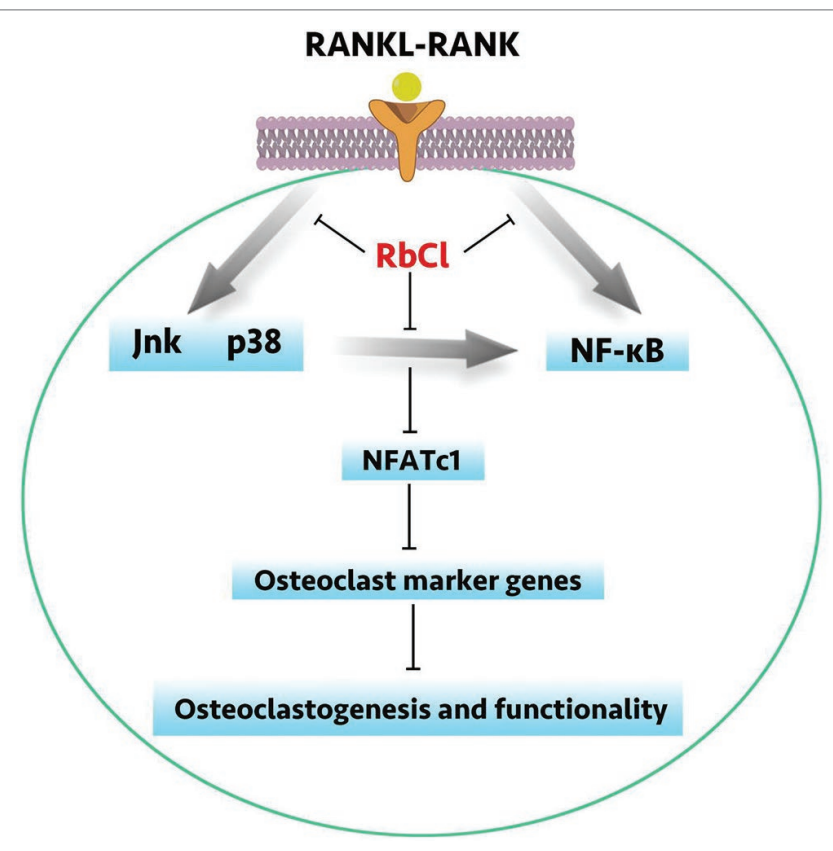

FIGURE 7 | A schematic diagram of $\mathrm{RbCl}$ in regulating osteoclastogenesis and osteoblastogenesis. By targeting Jnk and p38-mediated NF-kB activation, $\mathrm{RbCl}$ attenuated RANKL-induced expressions of osteoclast marker genes to inhibit osteoclastogenesis and functionality in vivo and in vitro. Additionally, $\mathrm{RbCl}$ could also enhanced ALP activity and mineralization in vivo and in vitro, thereby re-establishing the homeostasis of bone microenvironment to provide the potent possibility for future translational practice in clinic, such as the surface coating of orthopedic implant. possible future utilization of $\mathrm{RbCl}$ into surface coating of orthopedic implants to protect against osteoporosis.

In the recent times, osteoporosis has become a serious issue worldwide, which necessitates effective medical and economic remedies to improve the life quality and longevity of afflicted patients. Mainly two approaches, antiresorptive and anabolic reagents, have been deployed to treat osteoporosis (Amugongo et al., 2014). Representative candidates for antiresorptive reagents included bisphosphonate and denosumab. Although desirable effects of anti-osteoclastogenesis were shown after treatment with antiresorptive reagents, patients could suffer from inevitable side effects (Qiao et al., 2016), which led to the exacerbation of illness significantly. Besides, serving as anabolic reagents, teriparatide (recombinant human parathyroid hormone) and romosozumab enhanced bone mineral density significantly (Neer et al., 2001; Ishibashi et al., 2017). Nonetheless, their efficacy and safety still further require in-depth Phase III study (Liu et al., 2017). Previously, $\mathrm{RbCl}$ has been used as an effective non-invasive soluble biomarker to assess the perfusion ability of myocardial cells (Hougardy et al., 2003). Also, it elevates the production of nitric oxide to exert antidepressant effects (Kordjazy et al., 2015). No literature is concerned about the application of $\mathrm{RbCl}$ in orthopedic translational approach, which highlights its potential as a novel coating biomaterial in bone implants in future.

Importantly, in vitro results showed that $\mathrm{RbCl}$ not only inhibited the osteoclast formation and functionality but also facilitated osteoblast differentiation significantly. To be more specific, $\mathrm{RbCl}$ attenuated RANKL-induced formation of TRAPpositive osteoclast, F-actin ring, and bone resorption pits, whereas it promoted the intensities of ALP and ARS of MC3T3-E1 cells simultaneously. These results highlighted the combination of antiresorptive and anabolic effects by $\mathrm{RbCl}$, indicating a potent treatment strategy against osteoporosis.

As mentioned above, the process of osteoclastogenesis includes the activation of MAPK and NF- $\kappa$ B signaling. After the stimulation of RANKL, the extracellular stimulus was transferred into the nucleus for the upregulation of Jnk, p38, Erk, and NF- $\kappa \mathrm{B}$, resulting in the differentiation toward matured osteoclasts, with active expression of the osteoclast-specific genes CTR, Ctsk, NFATc-1, and TRAP. Herein, we found that $\mathrm{RbCl}$ was able to target NF- $\mathrm{BB}$ directly and indirectly. After the stimulation of Jnk/p38 activator and RANKL, RbCl inhibited the elevated expression of $\mathrm{p}-\mathrm{IKKa}$ and the degradation of $\mathrm{I} \kappa \mathrm{B} \alpha$ in osteoclast precursors, indicating indirect $\mathrm{NF}-\kappa \mathrm{B}$ inhibition via MAPK suppression. The resultant co-inactivation of MAPK and NF- $\kappa B$ by $\mathrm{RbCl}$ contributes to inhibit RANKLstimulated osteoclast formation synergistically, as further evidenced by the downregulated levels of osteoclastic genes and activity of NFATc-1, highlighting that MAPK/NF- $\kappa B$ targeting strategy may serve as a promising approach in the treatment of osteoporosis.

Furthermore, deficiency of estrogen could result in the advent of osteoporosis. Besides, wear particle (Ti) derived from prosthesis used during total joint arthroplasty (TJA) could recruit and activate osteoclasts to local sites, leading to undesirable prosthetic 
loosening (Ouyang et al., 2014). Hence, we used both OVX and $\mathrm{Ti}$ particles-associated calvaria osteolysis mice models to evaluate the in vivo osteoclast inhibitory effects of $\mathrm{RbCl}$ extensively. Our results demonstrated that $\mathrm{RbCl}$ is beneficial for the protection of trabecular microarchitecture by inhibiting TRAP-positive osteoclastogenesis in vivo. Taken together, these findings indicated that $\mathrm{RbCl}$ inhibited RANKL-induced osteoclast formation both in vitro and in vivo. However, details on osteoblast activity in vivo, mechanisms regarding osteoblastogenesis, the weight changes of uterus, side effects of other physiologic tissues and organs after $\mathrm{RbCl}$ treatments should be taken into future consideration, which would facilitate the clinical translational practice of $\mathrm{RbCl}$ against osteoporosis. Also, the molecular docking between $\mathrm{RbCl}$ and p38/Jnk was largely speculative, which required further biochemical analyses in-depth.

In conclusion, we found a brand-new application of $\mathrm{RbCl}$ in medical translation practice, i.e., suppression of osteoclastogenesis while facilitating osteoblastogenesis simultaneously via Jnk/p38-mediated NF- $\mathrm{BB}$ activation, both in vitro and in vivo. Our finding suggests the potential possibility of using $\mathrm{RbCl}$ in the future for surface coating of orthopedic biomaterial implants to protect against osteoporosis.

\section{ETHICS STATEMENT}

This study was carried out in accordance with the recommendations of guiding principles of Animal Care

\section{REFERENCES}

Amugongo, S. K., Yao, W., Jia, J., Dai, W., Lay, Y. A., Jiang, L., et al. (2014). Effect of sequential treatments with alendronate, parathyroid hormone (1-34) and raloxifene on cortical bone mass and strength in ovariectomized rats. Bone 67, 257-268. doi: 10.1016/j.bone.2014.04.033

Boyle, W. J., Simonet, W. S., and Lacey, D. L. (2003). Osteoclast differentiation and activation. Nature 423, 337-342. doi: 10.1038/nature01658

Chen, X., Zhi, X., Cao, L., Weng, W., Pan, P., Hu, H., et al. (2017). Matrine derivate MASM uncovers a novel function for ribosomal protein S5 in osteoclastogenesis and postmenopausal osteoporosis. Cell Death Dis. 8:e3037. doi: $10.1038 /$ cddis.2017.394

Dhawan, P., and Richmond, A. (2002). A novel NF-kappa B-inducing kinase-MAPK signaling pathway up-regulates NF-kappa B activity in melanoma cells. J. Biol. Chem. 277, 7920-7928. doi: 10.1074/jbc.M112210200

Hougardy, E., Pernet, P., Warnau, M., Delisle, J., and Grégoire, J.-C. (2003). Marking bark beetle parasitoids within the host plant with rubidium for dispersal studies. Entomol. Exp. Appl. 108, 107-114. doi: 10.1046/j.1570-7458.2003.00073.x

Ishibashi, H., Crittenden, D. B., Miyauchi, A., Libanati, C., Maddox, J., Fan, M., et al. (2017). Romosozumab increases bone mineral density in postmenopausal Japanese women with osteoporosis: a phase 2 study. Bone 103, 209-215. doi: 10.1016/j.bone.2017.07.005

Jiang, C., Xiao, F., Gu, X., Zhai, Z., Liu, X., Wang, W., et al. (2015). Inhibitory effects of ursolic acid on osteoclastogenesis and titanium particle-induced osteolysis are mediated primarily via suppression of NF-kappaB signaling. Biochimie 111, 107-118. doi: 10.1016/j.biochi.2015.02.002

Jing, H., Su, X., Gao, B., Shuai, Y., Chen, J., Deng, Z., et al. (2018). Epigenetic inhibition of Wnt pathway suppresses osteogenic differentiation of BMSCs during osteoporosis. Cell Death Dis. 9, 176-188. doi: 10.1038/ s41419-017-0231-0

Kordjazy, N., Haj-Mirzaian, A., Amiri, S., Ostadhadi, S., Kordjazy, M., Sharifzadeh, M., et al. (2015). Elevated level of nitric oxide mediates the
Committee of Central South University, and the protocol was approved by the Animal Care Committee of Central South University.

\section{AUTHOR CONTRIBUTIONS}

ZO carried out the molecular genetic studies, participated in the sequence alignment, and drafted the manuscript. QH and $\mathrm{BL}$ carried out the animal study. HW carried out the immunoassays and participated in the sequence alignment. YL participated in the design of the study and performed the statistical analysis. TL conceived of the study and participated in its design and coordination and helped to draft the manuscript. All authors read and approved the final manuscript.

\section{FUNDING}

This work was supported by the National Natural for Youths (Grant No. 81702670), the National Natural Science Foundation of China (Grant Nos. 81672176 and 81871783), the Natural Science Foundation of Hunan Province, China (Grant No. 2018JJ2565), the National Science Foundation for Post-Doctoral Scientists of China (Grant No. 2017M622601), and the National Natural Science Foundation for Distinguished Young Scholars (Grant No. 51625404).

anti-depressant effect of rubidium chloride in mice. Eur. J. Pharmacol. 762 411-418. doi: 10.1016/j.ejphar.2015.06.030

Korus, M., Mahon, G. M., Cheng, L., and Whitehead, I. P. (2002). p38 MAPKmediated activation of NF-kappaB by the RhoGEF domain of Bcr. Oncogene 21, 4601-4612. doi: 10.1038/sj.onc. 1205678

Liu, X., Chin, J. F., Qu, X., Bi, H., Liu, Y., Yu, Z., et al. (2017). The beneficial effect of praeruptorin $\mathrm{C}$ on osteoporotic bone in ovariectomized mice via suppression of osteoclast formation and bone resorption. Front. Pharmacol. 8:627. doi: 10.3389/fphar.2017.00627

Luo, X., Gu, S., Zhang, Y., and Zhang, J. (2018). Kinsenoside ameliorates oxidative stress-induced RPE cell apoptosis and inhibits angiogenesis via Erk/p38/NF-kappaB/VEGF signaling. Front. Pharmacol. 9:240. doi: 10.3389/ fphar.2018.00240

Neer, R. M., Arnaud, C. D., Zanchetta, J. R., Prince, R., Gaich, G. A., Reginster, J. Y., et al. (2001). Effect of parathyroid hormone (1-34) on fractures and bone mineral density in postmenopausal women with osteoporosis. N. Engl. J. Med. 344, 1434-1441. doi: 10.1056/nejm200105103441904

Ouyang, Z., Guo, X., Chen, X., Liu, B., Zhang, Q., Yin, Z., et al. (2018). Hypericin targets osteoclast and prevents breast cancer-induced bone metastasis via NFATc1 signaling pathway. Oncotarget 9, 1868-1884. doi: 10.18632/oncotarget.22930

Ouyang, Z., Tan, T., Liu, C., Duan, J., Wang, W., Guo, X., et al. (2019a). Targeted delivery of hesperetin to cartilage attenuates osteoarthritis by bimodal imaging with Gd2(CO3)3@PDA nanoparticles via TLR-2/NF-kappaB/ Akt signaling. Biomaterials 205, 50-63. doi: 10.1016/j.biomaterials.2019.03.018

Ouyang, Z., Wang, S., Zeng, M., Li, Z., Zhang, Q., Wang, W., et al. (2019b). Therapeutic effect of palbociclib in chondrosarcoma: implication of cyclindependent kinase 4 as a potential target. Cell Commun. Signal 17:17. doi: 10.1186/s12964-019-0327-5

Ouyang, Z., Zhai, Z., Li, H., Liu, X., Qu, X., Li, X., et al. (2014). Hypericin suppresses osteoclast formation and wear particle-induced osteolysis via modulating ERK signalling pathway. Biochem. Pharmacol. 90, 276-287. doi: 10.1016/j.bcp.2014.06.009 
Papa, S., Zazzeroni, F., Pham, C. G., Bubici, C., and Franzoso, G. (2004). Linking JNK signaling to NF-kappaB: a key to survival. J. Cell Sci. 117, 5197-5208. doi: 10.1242/jcs.01483

Qiao, H., Cui, Z., Yang, S., Ji, D., Wang, Y., Yang, Y., et al. (2017). Targeting osteocytes to attenuate early breast cancer bone metastasis by theranostic upconversion nanoparticles with responsive plumbagin release. ACS Nano 11, 7259-7273. doi: 10.1021/acsnano.7b03197

Qiao, H., and Tang, T. (2018). Engineering 3D approaches to model the dynamic microenvironments of cancer bone metastasis. Bone Res. 6, 3-15. doi: 10.1038/ s41413-018-0008-9

Qiao, H., Wang, T. Y., Yu, Z. F., Han, X. G., Liu, X. Q., Wang, Y. G., et al. (2016). Structural simulation of adenosine phosphate via plumbagin and zoledronic acid competitively targets JNK/Erk to synergistically attenuate osteoclastogenesis in a breast cancer model. Cell Death Dis. 7:e2094. doi: 10.1038/cddis.2016.11

Qu, X., Mei, J., Yu, Z., Zhai, Z., Qiao, H., and Dai, K. (2018). Lenalidomide regulates osteocytes fate and related osteoclastogenesis via IL-1beta/NFkappaB/RANKL signaling. Biochem. Biophys. Res. Commun. 501, 547-555. doi: $10.1016 /$ j.bbrc.2018.05.035

Rao, S. S., Hu, Y., Xie, P. L., Cao, J., Wang, Z. X., Liu, J. H., et al. (2018). Omentin-1 prevents inflammation-induced osteoporosis by downregulating the pro-inflammatory cytokines. Bone Res. 6, 9-21. doi: 10.1038/s41413-018-0012-0

Seeman, E., and Delmas, P. D. (2006). Bone quality--the material and structural basis of bone strength and fragility. N. Engl. J. Med. 354, 2250-2261. doi: 10.1056/NEJMra053077

Tan, H., Guo, S., Yang, S., Xu, X., and Tang, T. (2012). Physical characterization and osteogenic activity of the quaternized chitosan-loaded PMMA bone cement. Acta Biomater. 8, 2166-2174. doi: 10.1016/j.actbio.2012.03.013

Trott, O., and Olson, A. J. (2010). AutoDock Vina: improving the speed and accuracy of docking with a new scoring function, efficient optimization, and multithreading. J. Comput. Chem. 31, 455-461. doi: 10.1002/jcc.21334
Xiao, Z., Wan, J., Nur, A. A., Dou, P., Mankin, H., Liu, T., et al. (2018). Targeting CD44 by CRISPR-Cas9 in multi-drug resistant osteosarcoma cells. Cell. Physiol. Biochem. 51, 1879-1893. doi: 10.1159/000495714

Xiao, F., Zhai, Z., Jiang, C., Liu, X., Li, H., Qu, X., et al. (2015). Geraniin suppresses RANKL-induced osteoclastogenesis in vitro and ameliorates wear particle-induced osteolysis in mouse model. Exp. Cell Res. 330, 91-101. doi: 10.1016/j.yexcr.2014.07.005

Yang, S., Qiang, L., Sample, A., Shah, P., and He, Y. Y. (2017). NF-kappaB signaling activation induced by chloroquine requires autophagosome, p62 protein, and c-Jun N-terminal kinase (JNK) signaling and promotes tumor cell resistance. J. Biol. Chem. 292, 3379-3388. doi: 10.1074/jbc.M116.756536

Zhai, Z. J., Li, H. W., Liu, G. W., Qu, X. H., Tian, B., Yan, W., et al. (2014). Andrographolide suppresses RANKL-induced osteoclastogenesis in vitro and prevents inflammatory bone loss in vivo. Br. J. Pharmacol. 171, 663-675. doi: $10.1111 / \mathrm{bph} .12463$

Zhang, Q., Tang, X., Liu, Z., Song, X., Peng, D., Zhu, W., et al. (2018). Hesperetin prevents bone resorption by inhibiting RANKL-induced osteoclastogenesis and Jnk mediated Irf-3/c-Jun activation. Front. Pharmacol. 9:1028. doi: 10.3389/fphar.2018.01028

Conflict of Interest Statement: The authors declare that the research was conducted in the absence of any commercial or financial relationships that could be construed as a potential conflict of interest.

Copyright (c) 2019 Ouyang, Huang, Liu, Wu, Liu and Liu. This is an open-access article distributed under the terms of the Creative Commons Attribution License (CC BY). The use, distribution or reproduction in other forums is permitted, provided the original author(s) and the copyright owner(s) are credited and that the original publication in this journal is cited, in accordance with accepted academic practice. No use, distribution or reproduction is permitted which does not comply with these terms. 